\title{
The adsorption and inhibition mechanism of 1-Phenyltetrazole- 5-thiol for X70 steel corrosion in $\mathrm{H}_{2} \mathrm{SO}_{4}$ medium
}

\author{
Yanyan Xu ${ }^{1,2}$, Wenwen Xiao ${ }^{1,2}$, Pengli Ge ${ }^{1,2}$, Wenguang Zeng ${ }^{1,2}$, Qingshan Liu ${ }^{1,2}$, \\ Zhengyuan Gao ${ }^{3, *}$, Yongbo Yan ${ }^{4}$ \\ ${ }^{1}$ SINOPEC Northwest Company of China Petroleum and Chemical Corporation, Urumqi 830011, \\ China \\ ${ }^{2}$ Key Laboratory of Enhanced Oil Recovery in Carbonate Fractured-vuggy Reservoirs, SINOPEC, \\ Urumqi 830011, China \\ ${ }^{3}$ School of Mechatronics \& Vehicle Engineering, Chongqing Jiaotong University, Chongqing, \\ 400074, China \\ ${ }^{4}$ School of Oil and Natural Gas Engineering, Southwest Petroleum University, Chengdu 610500 \\ *E-mail: zhengyuangao@cqitu.edu.cn (Z.Gao); yanyongbo0520@163.com
}

Received: 5 August 2021 / Accepted: 18 September 2021 / Published: 10 November 2021

\begin{abstract}
This work aimed to develop a high-efficiency and reliable alternative for conventional and toxic corrosion inhibitor. A novel tetrazole derivative (1-Phenyltetrazole-5-thiol, PTT) that possessed excellent anti-corrosion capacity was exploited for the first time. Based on the traditional electrochemical investigation, morphological observation, and computer modeling, inhibition ability and adsorption type of PTT for X70 steel in $0.5 \mathrm{M} \mathrm{H}_{2} \mathrm{SO}_{4}$ were varied. The electrochemical results showed that PTT was a mixed organic inhibitor, which could retard charge transfer impedance, and slowed down the corrosion rate of steel. Specifically, the highest inhibition efficiency was $95.1 \%$ when the added PTT dose was $2 \mathrm{mM}$. The adsorption model showed that the PTT film was formed via powerful physicochemical adsorption. As shown in theoretical calculations, the lower $\Delta E$, higher $\mu$ values and

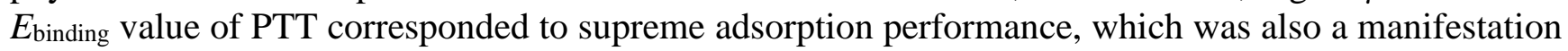
of the PTT high-efficiency corrosion inhibition.
\end{abstract}

Keywords: Corrosion inhibitor, Tetrazole derivative, Steel, Electrochemistry, Theoretical calculation

\section{$\underline{\text { FULL TEXT }}$}

(C) 2021 The Authors. Published by ESG (www.electrochemsci.org). This article is an open access article distributed under the terms and conditions of the Creative Commons Attribution license (http://creativecommons.org/licenses/by/4.0/). 